\title{
Philosophiques
}

\section{L'analogie et la vérité chez Franz Brentano}

\section{Marietje Van Der Schaar}

Volume 26, numéro 2, automne 1999

La critique de la raison en Europe centrale

URI : https://id.erudit.org/iderudit/004994ar

DOI : https://doi.org/10.7202/004994ar

Aller au sommaire du numéro

Éditeur(s)

Société de philosophie du Québec

ISSN

0316-2923 (imprimé)

1492-1391 (numérique)

Découvrir la revue

Citer cet article

Van Der Schaar, M. (1999). L'analogie et la vérité chez Franz Brentano. Philosophiques, 26(2), 203-217. https://doi.org/10.7202/004994ar

\section{Résumé de l'article}

Les idées aristotéliciennes sur l'analogie qui se trouvent dans la thèse de Brentano peuvent éclairer sa théorie de la vérité, tout comme ce qu'il écrit sur la notion de vérité à la fin de sa vie. Il semble que Brentano ne distingue pas la notion de la vérité et la notion d'évidence ; mais, à vrai dire, il a deux notions de vérité. Au sens originel, un jugement vrai signifie une évidence ; au sens figuré ou analogique, un jugement aveugle, qui correspond à un jugement évident sous tous les autres aspects, est aussi dit vrai. Ce jugement est vrai en tant qu'il peut être jugé avec évidence.
Ce document est protégé par la loi sur le droit d'auteur. L'utilisation des services d'Érudit (y compris la reproduction) est assujettie à sa politique d'utilisation que vous pouvez consulter en ligne.

https://apropos.erudit.org/fr/usagers/politique-dutilisation/ 


\title{
L'analogie et la vérité chez Franz Brentano
}

\author{
MARIETJE VAN DER SCHAAR \\ Université de Leiden, Pays-Bas \\ vdschaar@rullet.LeidenUniv.nl
}

\begin{abstract}
RÉSUMÉ. - Les idées aristotéliciennes sur l'analogie qui se trouvent dans la thèse de Brentano peuvent éclairer sa théorie de la vérité, tout comme ce qu'il écrit sur la notion de vérité à la fin de sa vie. Il semble que Brentano ne distingue pas la notion de la vérité et la notion d'évidence ; mais, à vrai dire, il a deux notions de vérité. Au sens originel, un jugement vrai signifie une évidence ; au sens figuré ou analogique, un jugement aveugle, qui correspond à un jugement évident sous tous les autres aspects, est aussi dit vrai. Ce jugement est vrai en tant qu'il peut être jugé avec évidence.
\end{abstract}

\begin{abstract}
Aristotelian ideas on analogy that may be found in Brentano's early thesis help to elucidate his theory of truth, especially what Brentano wrote on truth at the end of his life. It seems as though Brentano did not distinguish between the notion of truth and the notion of evidence ; but, in fact, he has two notions of truth. The original sense of truth in a true judgment means evidence ; in a derived or analogical sense, a blind judgment, which corresponds to an evident judgment in all other aspects, is also called true. Such a judgment is true in so far as it may be judged with evidence.
\end{abstract}

\section{Introduction}

Le livre le plus connu de Brentano est intitulé Psychologie du point de vue empirique. La psychologie dont il s'agit là n'est pas une psychologie empirique au sens moderne du mot. Brentano qualifie sa psychologie de « descriptive ». Ce qu'il décrit sont les phénomènes psychiques qui nous sont donnés par la perception interne. Q uelquefois, Brentano nomme sa psychologie «psychologie analytique », car la psychologie analyse les phénomènes psychiques jusque dans les éléments les plus petits, comme nous décomposons les mots en lettres. Dans cette psychologie descriptive, Brentano prête attention aux notions de base de la psychologie, qui, pour lui, sont aussi les notions de base de la philosophie. Entre la publication de cette œuvre en 1874 et l'œuvre suivante d'importance, L'origine de la connaissance morale, il y a quinze ans. Q uoiqu'il n'ait rien changé à son projet, la question centrale que se pose alors Brentano est la suivante : comment une psychologie descriptive peut-elle fournir un fondement aux sciences normatives que sont la logique et l'éthique?

Bien quel'on puisse dire que Brentano est un « empiriste » en tant qu'il croit que tous nos concepts ont leur origine dans l'expérience, il n'est pas un empiriste au sens où il croit que la tâche de l'éthique et de la logique consiste seulement à décrire ce que nous jugeons comme bon, ou ce que nous jugeons comme vrai ou valide. II estime aussi que le domaine des vérités nécessaires est plus large que celui des mathématiques et de la logique. Bien que Bren- 
tano ait subi l'influence de Stuart $M$ ill et de Comte, il a donné une interprétation propre à l'empirisme. II ne faut pas oublier que Brentano était prêtre et qu'en tant que tel, il avait pris connaissance de la scolastique, surtout de I'œuvre de saint Thomas. Un autre élément important pour son développement fut le regain d'intérêt pour A ristote au dix-neuvième siècle, que l'on peut voir, par exemple, dans l'œuvre de Trendelenburg, qui fut l'un des maîtres de Brentano et écrivit sur la doctrine des catégories chez A ristote. Et c'est cette doctrine qui constitue aussi le sujet de la thèse de Brentano.

$D$ ans cet article, je veux montrer que les idées aristotéliciennes sur I'analogie qui se trouvent dans la thèse de Brentano peuvent éclairer sa théorie de la vérité, non seulement sa théorie de la vérité comme il la défend avant 1890, mais aussi ce qu'il écrit sur la notion de vérité à la fin de sa vie, dans les années 1914 à 1916. Cette théorie de la vérité des années 1914-1916 est difficile à interpréter, parce que Brentano utilise différentes notions de vérité côte à côte.

En ce qui concerne l'ontologie de Brentano, une distinction entre ses écrits antérieurs et ses écrits postérieurs est très éclairante. La notion de I'analogie joue un rôle important dans l'ontologie du « jeune » Brentano: l'étant est analogue, c'est-à-dire que l'être a plusieurs formes. D ans les écrits du « vieux » Brentano, la thèse défendue est que l'être n'a qu'une seule forme : l'être individuel. L'être n'est pas analogue, mais univoque. En ce qui concerne son épistémologie, je crois qu'il y a plus de continuité dans la pensée de Brentano. Le « jeune » Brentano utilise la notion d'analogie pour mettre en relation l'une avec l'autre les différentes notions de la vérité ; il fait la même chose dans ses écrits postérieurs, bien qu'il n'utilise plus le mot « analogie».

La théorie de la vérité de Brentano est d'importance parce qu'il s'efforce de dével opper un concept de vérité non relativiste sans pour autant supposer l'existence de vérités ontologiques, de vérités indépendantes de nos jugements. Brentano veut également présenter une notion de vérité qui nous fournisse un critère nous permettant de déterminer si nos jugements sont vrais ou faux.

II existe différentes possibilités d'éclairer la notion de vérité au moyen de la notion de l'analogie. Dans le paragraphe 1 , je veux montrer que Brentano croit qu'il y a un parallèle substantiel à faire entre la notion du vrai et celle du bien. II y a une égalité de rapports - une analogie au sens strict entre ces deux notions.

La thèse de Brentano, publiée en 1862, traite du problème que l'on rencontre chez A ristote et qui consiste à tenter de comprendre comment il est possible de dire « être » de choses de types très divers. La question qui se pose est : est-ce par hasard que nous utilisons le mot «être » de façons si diverses? O u y a-t-il une cohérence à la base de ces usages divers? Est-il possible de relier les sens divers du mot « être » à un sens fondamental? En ce cas, on parle d'une analogie par rapport à l'Un (comme je l'ex pliquerai dans le paragraphe 2). 
N ous pouvons nous poser une question semblable à l'égard de l'usage du terme « vrai ». De même qu'il y a, pour A ristote, un seul sens originel du terme « être », de même y a-t-il, pour Brentano, un seul sens originel du terme « vrai ». Je reviendrai sur ce point dans les paragraphes 3 et 4 .

\section{\1 L'analogie entre la notion du vrai et celle de bien}

Q uand A ristote emploie le terme « analogie », il parle toujours d'une égalité de rapports. En premier lieu, il emploie la notion d'analogie en biologie pour souligner la ressemblance des fonctions des organes d'animaux de diverses espèces. II l'emploie aussi pour montrer que les axiomes et les preuves mathématiques s'appliquent à des sortes de choses diverses comme les points, les lignes, les moments et les corps ${ }^{1}$. C'est ainsi qu'A ristote rend plausible l'existence de jugements généraux sur des choses de sortes très diverses. Les sciences comme la biologie exigent une unité du genre à l'égard des objets auxquels elles s'appliquent. N éanmoins, il est aussi possible qu'une science n'ait qu'une unité plus faible que celle du genre ou genus, celle de l'analogie? Dans ce sens, les mathématiques sont un exemple pour la philosophie de I'être d'A ristote. À la fin de la M étaphysique, A ristote tire une conclusion importante pour la possibilité d'une philosophie de l'être : il y a une unité d'analogie entre les catégories différentes ${ }^{3}$.

A ristote dit à propos de l'unité d'analogie qu'elle existe quand une première chose est dans un rapport à une deuxième chose comme une troisième à une quatrième ${ }^{4}$; il y a une ressemblance de rapports. Un exemple connu de cette analogie vient de l'Éthique à N icomaque : " l'intelligence est à l'âme, comme le visage est au corps $»^{5}$. Au moyen de cette analogie, on peut dire quelque chose sur les notions indéfinissables comme les notions de base que sont l'être, le bien et le vrai ${ }^{6}$.

Les scolastiques ont utilisé systématiquement cette forme d'analogie sous le nom d'analogie proportionnelle (analogia proportionalitas). Selon saint Thomas, nous pouvons dire quel que chose sur la connaissance de Dieu, bien que nous ne puissions rien savoir sur Dieu même. La connaissance de Dieu est à Dieu dans le même rapport que la connaissance de l'homme à I'homme ${ }^{7}$. II n'est aucunement question d'une réduction de l'un à l'autre. Dans le cas de cette forme d'analogie, les termes de la comparaison et ceux des rapports comparés gardent leur indépendance. Suivant l'exemple de saint Thomas, Brentano parle d'«A nalogie der Proportionalität». N ous disons qu'un monarque est « roi » par rapport aux autres humains, que

1. A ristote, Seconds A nalytiques, 74a17-25.

2. A ristote, M étaphysique, 1017a2.

3. Ibid., $1093 b 18$.

4. Ibid., $1016 \mathrm{~b} 31$.

5. A ristote, E thique à $\mathrm{N}$ icomaque, $1096 \mathrm{~b} 28$.

6. Aristote, M étaphysique, $1048 a 35$ et suivantes.

7. Saint Thomas, D e Veritate, II,11,C., Klubertanz, 1960, p. 190, 89 
l'aigle est « roi » par rapport aux autres oiseaux. D e même nous parlons du roi au jeu d'échecs. D ans tous ces cas il y a une analogie, c'est-à-dire une égalité de rapports, une parenté de concepts ${ }^{8}$.

En philosophie, il n'est pas inhabituel de comparer les notions du vrai et du bien. M ais la comparaison ne va pas de soi dans la tradition aristotélicienne, parce que pour A ristote, la vérité revient premièrement à l'intelligence, alors que le bien se trouve dans les choses.

Déjà dans la Psychologie de 1864, Brentano établit une comparaison entre les objets de la logique, les jugements, et les objets de l'éthique, les émotions, qui comprennent les actes de la volonté (voluntates sive affectus) ${ }^{9}$. Chacune des deux sortes de phénomènes psychiques, les jugements et les émotions, forme un domaine indépendant. En même temps, ces deux sortes de phénomènes montrent des ressemblances importantes. J ugements et émotions sont en effet tous deux bâtis sur des représentations ${ }^{10}$. Et, contrairement aux représentations, les jugements comme les émotions se trouvent en deux qualités différentes : tout jugement est une acceptation ou un rejet, toute émotion est un amour ou une haine ${ }^{11}$. L'une des deux qualités est positive (l'affirmation, l'amour), l'autre négative. II faut faire attention à ne pas pousser trop loin cette comparaison. Dans la Psychologie, Brentano dit que les deux sortes de phénomènes manifestent des degrés d'intensité, mais plus tard il retire cette thèse ${ }^{12}$. Car s'il y a un « meilleur » et un « moins bon » dans le domaine des émotions, il n'y a pas de «plus vrai » et de «moins vrai » en matière de jugement.

Dans L'origine de la connaissance morale de1889, Brentano donne la priorité à l'analogie entre l'éthique et la logique. II compare l'éthique à la logique pour rendre plausible l'objectivité de l'éthique ${ }^{13}$. Le problème de Brentano est de savoir comment critiquer le naturalisme et I'historisme sans être obligé de supposer quele bien soit une qualité objective. Cen'est pas tant la notion de la qualité objective du bien mais la notion de la correction ou de

8. Brentano, 1960, p. 93. Je laisse de côté la distinction que fait Brentano entre deux formes del'analogie proportionnelle. Brentano appelle la forme dont il s'agit ici « qualitative »: différentes qualités se rapportent de la même façon à plusieurs sujets. Seule cette forme est d'importance pour sa philosophie.

9. Déjà dans sa thèse, Brentano attire l'attention sur la ressemblance entre le bon et le vrai : le bon est la fin de la volonté ; le vrai est la fin de l'intelligence: «Das Gute is das, was der Wille erstrebt, so ist das Wahre das, worauf der Verstand als sein Ziel gerichtet ist. « (Brentano, 1960, p. 29).

10. Brentano, 1874, p. 291.

11. I bid., p. 263, 292.

12. Brentano, 1911, p.142.

13. Dans son article sur la vérité de 1889, Brentano éclaire la notion de base de la logique à l'aide de la notion de base de l'éthique. De même dans ses écrits postérieurs, il soutient cette thèse de l'analogie entre les deux domaines, par exemple dans « Seiend, Wahr und Gut » (3-51908), Brentano, 1966, p. 357-359 (le manuscrit M 38), et dans une lettre à Kraus du 14 septembre 1909 (Brentano, 1966, p. 207). Chaque fois, la comparaison n'est pas poussée trop loin. 
la justesse des émotions qui prend une place centrale dans l'éthique de Brentano. De deux émotions contraires, l'une est correcte, l'autre incorrecte ${ }^{14}$. « N ous disons que quelque chose est vrai lorsque l'acceptation qui s'y rapporte est juste. $\mathrm{N}$ ous disons que quelque chose est bien lorsque l'amour qui s'y rapporte est juste. ${ }^{15}$ Le discernement de ce qui est digne de notre amour est l'analogon de l'évidence dans le domaine du jugement ${ }^{16}$. II n'y a pas que les jugements, mais aussi les émotions qui sont susceptibles d'être dites justes ou non ${ }^{17}$.

N ous disons que le jugement ou l'émotion de quelqu'un est juste, quand celui ou celle-ci convient, est en harmonieavec les objets ${ }^{18}$. Direqu'un objet est vrai ou bon ne signifie cependant rien d'autre que dire que l'acceptation ou l'amour de cet objet est juste. $\mathrm{N}$ ous ne pouvons pas éprouver la vérité ou la bonté d'un objet extérieur à nous; par contre, nous pouvons éprouver la justesse d'une émotion ou d'un jugement. Brentano définit donc les notions du vrai et du bon en termes de justesse (voir paragraphe 4). Dans les jugements comme dans les actes moraux, il y a une préférence d'ordre intérieur qui distingue la volonté morale de la volonté immorale et différencie le jugement vrai du préjugé ${ }^{19}$. Les deux sortes de phénomènes réveillent une certaine justesse intérieure, de sorte que l'éthique et la logique comportent un élément normatif inhérent aux phénomènes psychiques mêmes.

\section{đ2 L'analogie par référence à l'Un}

Signaler une égalité de rapport n'a qu'une valeur relative quand nous vouIons savoir quel que chose sur les termes de ce rapport. Dire que le vrai est par rapport au jugement juste comme le bien par rapport à l'émotion juste montre quel que chose des notions de base en général et souligneque celles-ci sont en rapport à l'expérience, mais ne dit pas grand-chose de la notion du vrai en particulier. $\mathrm{N}$ ous voyons un problème comparable dans la $\mathrm{M}$ étaphysique d'A ristote : la thèse selon laquelle il y a une égalité de rapports entre les catégories différentes ne montre pas encore qu'une philosophie de l'être soit possible. Celle-ci n'est possible que si toutes les manières d'être peuvent être ramenées à une seule. Cette manière d'être qui est à la base des autres est la manière d'être de la substance (« ousia »). Q ue nous puissions dire qu'une longueur est de deux mètres, cela est fondé sur l'être de la substance à laquelle cette longueur revient. « Être » est dit des différentes catégories par

14. Roderick Chisholm montre que, dans ses écrits postérieurs, Brentano a supprimé cette forme d'analogie entre les jugements et les émotions (Chisholm, 1982, p. 77 ; cf. Chisholm, 1986, ch. 4).

15. Brentano, 1889, p. 17[19].

16. Ibid., p. 21[22].

17. Brentano, 1911, p. 140, Brentano, 1874, p. 312.

18. Brentano, 1889, p. 76 [60].

19. Ibid., p. 11[13]. 
référence à I'Un ( « pros hen »), à une seule nature (« archè ») ${ }^{20}$. Et la manière dont les différentes formes d'être sont en rapport avec cette forme d'être originelle de la catégo rie de la substance détermine les catégories à distinguer ${ }^{21}$. Bien que la division des catégories prenne son point de départ dans des considérations grammaticales, le but est d'en arriver, par l'analyse des structures logiques sous-jacentes, à des distinctions ontologiques.

A ristote n'utilise pas le mot « analogie » pour désigner le rapport à I'Un, alors que la tradition scolastique introduit cet usage : elle emploie le terme « analogie attributive» (analogia attributionis) ${ }^{22}$. Brentano dit : «A nalogie zum gleichen Terminus », c'est-à-dire, " analogie au même terme », ou même «terminus ». Selon la scolastique, les termes d'une relation sont des objets reliés; le « terminus » d'un rapport est ce à quoi l'autre terme (la base) est relié. « Terme » ou « terminus » ne doivent pas être entendus comme des éléments linguistiques. Le terminus est ce que « vise » l'autre terme. Brentano donne la priorité à cette analogie dans sa thèse, et cherche à justifier la division des catégories aristotéliciennes ${ }^{23}$.

L'exemple classique, empruntéà A ristote, d'un mot qui est analogue par référenceà l'Un est le mot « santé ». " Sain » se dit d'abord del'état d'un organisme, puis du remède à titre de cause productrice, ou del'urineà titre de signe. Brentano donne deux exemples. Les mots « heilsam » et « heilbar » ont une même source, comme les mots « étranger » et « étrange » en français, et c'est pourquoi on les dit « analogues par référenceà l'Un ». A ristote appelle les choses indiquées par ces mots des « paronymes » 24 . Un mot comme « royal » est aussi analogue par référence à I'Un, parce que ses différents sens se groupent autour de l'un d'entre eux. Brentano appelle ces termes des «noms de famille ${ }^{25}$. N ous parlons par exemple non seulement de souverain royal, le détenteur du pouvoir royal, mais aussi de sceptre royal, d'honneur royal, d'ordonnance royale, de sang royal. O n appelle cet honneur « royal » parce que c'est I'honneur du souverain royal. Dans tous ces cas d'analogie par référence à l'Un, nous pouvons distinguer entre l'usage primaire et l'usage secondaire d'un terme : sel on la définition et selon la connaissance.

20. A ristote, M étaphysique, $1003 \mathrm{~b} 6$.

21. Ibid., 1061a11.

22. Q uelques-uns, comme $\mathrm{O}$ wen, refusent par conséquent d'appeler « analogie » cette forme de polysémie chez A ristote. O wen emploie leterme « focal meaning » (lefoyer), termequi est un peu trompeur parce que, chez A ristote, il ne s'agit pas des sens du terme être, mais des manières d'être mêmes (O wen, 1960,p. 180 ; cf. Kahn, 1978, p. 264).

23. Brentano, 1960, p. 109, 113.

24. Aristote, Catégories, 1 la12 et suivantes. II n'est pas correct de dire que les termes « paronyme » et « analogue par référence à l'un » sont synonymes; dire de quelque chose ou de quelque mot que ce sont des paronymes est une caractérisation grammaticale.

25. Brentano, 1960, p. 96 . II y a clairement une différence avec la notion de Familienähnlichkeit utilisée par W ittgenstein. Ce dernier montre, lui aussi, que les objets qui sont indiqués par un mot ne doivent pas avoir un noyau fixe de caractéristiques. $M$ ais Brentano croit à l'existence d'un sens primaire, un « père » pour ainsi dire. 
Les termes qui sont analogues par référence à I'U n sont-ils plus près des termes univoques que les termes qui sont analogues au sens strict (ceux avec une égal ité de rapports), puisqu'on peut réduire les sens différents à un sens fondamental? Ce qui n'est pas substance est plutôt d'un être (génitif), qu'un être lui-même ${ }^{26}$. C'est pourquoi nous pouvons dire que le terme « être » est aussi univoque à certains égards ${ }^{27}$. Bien que le sens primaire ne soit pas universellement présent dans toutes les manières d'être, c'est sur le sens central qu'une philoso phie del'être se concentre principalement. N ous pouvons dire que l'être substance est le sens propre du mot « être » ou la vraie manière d'être. «Propre» (« eigentlich ») est la traduction donnée par Brentano du mot grec « haploos », quel'on peut aussi traduire par « absolu », « simple», «non accidentel » ou « sans qualifications ${ }^{28}$.

Dans ses écrits postérieurs, Brentano insiste sur la distinction entre I'usage propre et l'usage impropre d'un terme comme « être » ${ }^{29}$. Cette distinction correspond à ce que nous pouvons nous représenter comme existant indépendamment, et ce que nous ne pouvons pas nous représenter ainsi. $\mathrm{N}$ ous ne pouvons nous représenter que les substances. À ce stade de sa pensée, Brentano dit que seuls les objets individuels sont vraiment. En ce sens, Brentano est dit réiste.

Tant que l'on ne s'imagine pas qu'un terme qui est utilisé improprement signifiequelque chose de réel, cet usage peut être particulièrement utile. Par exemple, selon Brentano, nous employons souvent en arithmétique des chiffres qui n'indiquent pas des nombres, parce que nous ne pouvons pas nous représenter ces nombres. Les chiffres avec lesquels nous calculons sont seulement des succédanés : ils ne sont que des signes ou symboles (ens linguae) qui servent de moyen ${ }^{30}$. L'objet de ce penser est un signe qui est un ersatz de vrai nombre. Cela n'empêche pas que nous puissions obtenir des vérités en calculant avec de tels chiffres, parce qu'il y a un rapport systématique entre les signes et ce qui est en réalité. Puisque l'usage impropre d'un terme est très utile, comme Brentano l'admet, il est préférable de parler de l'usage d'un terme absolu, simple ou sans qualifications, à la différence de I'usage d'un terme avec qualifications, au sens modifié.

\section{\3 La vérité revient en premier lieu aux jugements}

D equelle façon l'idée d'une analogie par référenceà l'Un peut-elle nous aider à mieux comprendre la théorie de la vérité de Brentano? C ette forme d'ana-

26. Ibid., p. 219.

27. Ibid., p. 97, 147, A ristote, M étaphysique, $1003 \mathrm{~b} 15$.

28. Brentano, 1960, p. 97 ; A ristote, M étaphysique, 1028a31, $1005 a 7$.

29. Brentano, 1974, p. 78 et suivantes; Brentano, 1985, p. 3 et suivantes.

30. Brentano, 1974, p. 92 (1906), H usserl, 1994, p. 32, Brentano, 1974, p. 155 (1905), Brentano, 1956, p. 27 (le manuscrit EL 72/le typoscrit EL 80 (1880-1890)). Je remercie la Direction des Archives H usserliennes de Louvain de m'avoir donné accès aux microfiches qui contiennent les copies des manuscrits de Brentano et des textes typographiés de ces manuscrits. 
logie nous fait comprendre qu'il y a un rapport systématique entre les différents sens du terme de vérité tel que Brentano l'utilise. "Tout comme le nom de l'étant [...] le mot "vrai" est équivoque, et est dit de l'un et de l'autre de façon homonyme [...] ces choses différentes ne sont pas dites vraies [...] de la même manière, mais de manière analogique ([...] pros hen) », écrit Brentano dans sa thès $\mathrm{e}^{31}$. D ans son article sur la vérité de 1889, « Über den Begriff der Wahrheit », il défend aussi ce rapport systématique, mais il n'utilise pas le mot « analogie». Et dans les manuscrits de 1914 et 1915, nous pouvons aussi trouver la comparaison entre le mot « vrai » et le mot « sain », mais sans le mot « analogie $» 32$.

Le terme « vrai » a un premier sens quand il est dit de l'intelligence jugeant ou d'un jugement (1); puis, des affirmations peuvent être dites « vraies» (2); « vrai » a encore un autre sens quand « vrai » est dit des représentations et des contenus des actes psychiques (3), et encore un autre quand il est dit des choses (4). Toutes ces différentes choses sont dites vraies de façon analogique par référence à I'Un ${ }^{33}$; et I'Un auquel les autres sont référés est la vérité du jugement.

La pensée qu'il y a différents usages du mot vérité et que tous les usages peuvent se ramener à la vérité du jugement se trouve aussi chez A ristote. Saint Thomas soutient une thèse semblable à celle d'A ristote: "La vérité revient premièrement à l'intelligence, secondairement aux choses » (« veritas per prius est in intellectu, et per posterius in rebus ${ }^{34}$ ). Une inversion des priorités est aussi défendue. Pour Bolzano, qui distingue aussi différents sens du mot « vérité », le sens premier et le plus originel de ce mot est la vérité qui revient aux propositions objectives ${ }^{35}$, puisque la vérité est contraire à la fausseté seulement dans les propositions ; dans les jugements, la vérité est contraire à l'erreur.

ad 1. Pour Brentano, la vérité revient donc en premier lieu aux jugements justes. $D$ ans sa théorie, vérité et fausseté signifient justesse et erreur ${ }^{36}$. C'est de ce premier sens du « vrai » dont il s'agit dans le paragraphe suivant.

ad 2. C'est pourquoi une affirmation qui exprime un jugement correct est dite « vraie».

ad 3. Une représentation, qui est pour Brentano un acte, peut être dite vraie ou fausse, parce qu'elle donne lieu à un jugement vrai ou faux. Par exemple, une hallucination est dite fausse en ce sens. Selon Brentano, les concepts sont

31. Brentano, 1960, p. 25.

32. «In ähnlicher Weise wie das Wort «gesund» ist auch das Wort «wahr» mehrdeuting, wobei wie dort auch hier eines ist, was im eigentlichen Sinne so hei $\beta t$, während alles andere nur wegen seiner Beziehung zu jenem so genannt wird. Dort der Leib, hier das Urteil. » (Brentano, 1970, p. 149, typoscrit EL 96, p.5).

33. Brentano, 1960, p. 25 et suivantes, Brentano, 1974, p. 6.

34. Saint Thomas, Summa Theologiae, q.16, a.6 ; cf. a.1, ad 3. ; saint Thomas, De Veritate, q.1.a.2.

35. WL, § 24.

36. Brentano, 1960, p. 31 ; Brentano, 1974, p. 6. 
une espèce spéciale de représentations. Les concepts qui contiennent une contradiction peuvent être dits faux, parce que quelqu'un qui croit que ces concepts s'appliquent à quelque chose juge faussement. En fait, tout concept peut être dit faux par rapport à l'un ou l'autre : le concept de la figure quadrangulaire est faux en tant qu'il ne recouvre pas le contenu du vrai concept du carréén.

Pour A ristote, comprendre peut être « vrai » en un autre sens ${ }^{38}$. II veut dire que les objets métaphysiques, comme $D$ ieu, qui sont simples, ne peuvent pas être jugés, mais seulement « compris », « aperçus » ou « touchés », et toucher de tels objets est toujours les comprendre comme ils sont en réalité. On appelle cela la vérité ontologique ${ }^{39}$. Le contraire de la compréhension vraien'est pas la fausseté ou l'erreur, mais la non-compréhension. Pour Brentano cependant, qui pense que l'objet du jugement n'a pas à être composé, apercevoir un objet de cette façon est un jugement par excellence.

Le contenu d'un jugement juste peut aussi être dit vrai. Comme juger est, pour Brentano, affirmer ou nier un objet, le contenu d'un jugement est : que l'objet existe ou que l'objet n'existe pas. Dans les termes utilisés par Brentano en 1889, le contenu est l'existence d'un objet ou la non-existence d'un objet. Q uand l'affirmation ou la négation d'un objet est juste, on peut aussi dire : I'existence ou la non-existence de cet objet est, ou est vraie. Ici, être signifie être vrai ${ }^{40}$. Implicitement, Brentano soutient la thèse qu'il y a des propositions, mais il ne dit pas que les propositions sont des réalités indépendantes. La justesse d'une affirmation et l'être-vrai de l'existence del'objet affirmé sont des corrélatifs au sens où l'un(e) ne peut pas exister sans l'autre ${ }^{41}$. La proposition n'existe que pour autant qu'il existe un certain jugement, un certain acte de juger. II en va de même pour la fausseté. La fausseté du jugement «A est » (il y a des licornes) et lenon-être de l'existence d'A (des licornes) sont aussi des corrélatifs. En ce sens, une fausseté est le contenu d'un jugement faux ${ }^{42}$. En fait, l'être comme être vrai ou faux est une forme d'être immanente ou intentionnelle, une idée qui se trouve déjà dans la thèse de Brentano ${ }^{43}$. O n peut dire que « vrai » dans «l'être vrai » modifie le sens du mot « être».

ad 4. Un objet peut aussi être dit vrai en tant qu'il peut êtrel'objet d'une affirmation juste. II n'est pas nécessaire d'affirmer l'objet de fait pour le dire vrai.

37. Cf. A ristote, M étaphysique, delta, $1024 b 25$ et suivantes.

38. A ristote, M étaphysique, theta, ch. 10.

39. C'est pourquoi l'on dit qu'A ristote défend non seulement une vérité du jugement, ou logique, mais aussi une vérité ontologique. Paul Wilpert le défend, mais il affirme aussi que cette vérité est une vérité impropre, le résultat d'une transmission conceptuelle par analogie (cf. Wilpert, 1972, p. 117).

40. Cf. Brentano, 1960, p. 33, 34.

41. Brentano, 1974, p. 27, 31.

42. Cf. A ristote, M étaphysique, 1017a31et suivantes, et ibid., E 4, $1027 \mathrm{~b} 17$ et suivantes.

43. Brentano, 1960, p. 37, 202. II utilise la terminologie de la scolastique: « Gedankending [...] objectiv in unserem Geiste existirend » (ibid., p. 37). 
En tant que l'objet est à affirmer, il est vrai. N ous parlons d'un Dieu vrai, parce que quelqu'un qui croit qu'il existejuge correctement ${ }^{44}$. $\mathrm{N}$ ous pouvons aussi dire qu'un objet existant est « faux», par exemple un rêve, lorsque nous sommes tentés d'en juger faussement. Et un homme est appelé « faux » $s^{\prime} i l$ est menteur ${ }^{45}$.

« Vrai » se dit aussi des objets au sens où le vrai est le contraire de l'apparent. Un ami vrai est un ami commeil doit être, contrairement à un ami apparent, dit Brentano. $\mathrm{N}$ ous pouvons trouver cette forme de vérité chez saint Anselme, qui défend une notion de vérité comme rectitude ${ }^{46}$. O n parle de la vérité d'objet, ou mieux, de la vérité de norme. Selon saint Anselme, (l'essence d')un objet est vrai, s'il est comme il est dans la pensée de Dieu ( «D e Veritate essentiae rerum ${ }^{47}$ ). L'idée de la vérité de l'objet est reprise par saint Thomas, mais avec cette différence qu'il la met en deuxième place. $L$ a vérité comme norme, tout commela vérité ontologique d'A ristote, $n$ 'a pas de contraire. Q uoique, pour Brentano, cette forme de vérité soit secondaire, elle peut aider à éclairer sa théorie de la vérité.

\section{$\int 4$ La vérité comme évidence versus la vérité comme évidentiabilité}

Comme pour A ristote, il n'est pas possible pour Brentano de définir la notion de vrai, parce que cette notion est élémentaire ${ }^{48}$. $0 \mathrm{n}$ peut cependant éclairer cette notion non seulement par une comparaison avec la notion de bien, mais aussi en considérant des exemples de jugements justes.

$M$ ais pourquoi Brentano soutient-il la thèsequela véritérevient en premier lieu au jugement? Selon Brentano, tous les concepts trouvent leur origine dans l'expérience, même les notions abstraites comme la vérité. $0 \mathrm{n}$ doit l'avoir vue ou vécue, de même que pour savoir ce qu'est un jugement, on doit avoir soi-même jugé ${ }^{49}$.

La notion de vérité peut se comprendre en considérant la distinction entre un jugement évident et un jugement aveugle. Supposez que vous désiriez quelque chose et que vous jugiez que votre désir existe. Brentano dit que ce jugement est prononcé avec évidence. Supposez maintenant que vous expliquiez à quelqu'un votre désir et que celui-ci juge aussi que votre désir existe. Cette autre personne ne peut pas prononcer ce jugement avec évidence, c'est pourquoi Brentano l'appelle « jugement aveugle». En comparant un jugement évident avec un jugement aveugle, nous pouvons voir ce qu'est l'évidence.

44. Brentano, 1974, p. 6 ; cf. Brentano, 1960, p. 31,32 et Brentano, 1889, p. 75 [59].

45. Brentano, 1960, p. 32 ; cf. Aristote, Métaphysique, delta, ch.29, $1024 \mathrm{~b} 17$ et suivantes.

46. Saint Anselme, D e Veritate, ch. II.

47. I bid., ch. VII.

48. Brentano, 1970, p. 149.

49. Ibid., p. 149. 
$M$ ais vous vous demanderez : quelle relation y a-t-il entre l'évidence et la vérité? Pour Brentano, « jugement évident » et « jugement vrai » signifient la même chose ${ }^{50}$. Comme la vérité, l'évidence ne connaît pas de degrés. En 1889, Brentano dit qu'on ne peut pas distinguer l'évidence d'un jugement de sa vérités1. Selon Brentano, l'évidence n'est pas un sentiment de contrainte intérieure; ce n'est pas l'impossibilité de douter de quelque chose, puisque mes préjugés sont pour moi aussi indubitables ${ }^{52}$. Q ue l'évidence soit inséparable de la vérité signifie que si nous jugeons avec évidence, nous ne pouvons pas nous tromper. L'évidence n'est aucunement quelque chose de subjectif ; quelqu'un qui juge avec évidence s'aperçoit en même temps que son jugement a une validité universelle. Cela veut dire que ce qui est affirmé par quelqu'un avec évidence ne peut être nié avec évidence par quelqu'un d'autre. De deux jugements contraires, un seul peut être jugé avec évidence ${ }^{53}$. O utre les perceptions internes, les axiomes, par exemple les axiomes mathématiques, sont immédiatement évidents. Les axiomes ou les jugements analytiques sont intuitionnés ex terminis ${ }^{54}$.

Le jugement évident s'accompagne d'une clarté propre. Être «clair » n'est pas la même chose qu'être « distinct ». J e peux par exemple très bien saisir que je désire quelque chose sans saisir distinctement ce que je désire. Brentano, fidèle à la tradition cartésienne, croit que la connaissance est toujours connaissance sûre. S'il arrive que je me trompe, c'est que je crois que mon jugement est évident alors que mon jugement n'est évident qu'en apparence. La fausseté d'un jugement ne peut être décelée qu'indirectement : si votre jugement contredit le mien, qui est évident, je peux juger avec évidence que votre jugement est faux ${ }^{55}$.

Un jugement évident n'a pas besoin de critère : il est sa propre mesure ${ }^{56}$. Les jugements qui en découlent, les jugements évidents indirects, ont des jugements évidents directs pour fondement, de sorte que ces derniers constituent bien un critère pour les jugements évidents indirects.

Brentano défend une théorie de l'évidence de la vérité parce qu'il veut donner une théorie de la vérité épistémologique. II critique la définition de la vérité comme correspondance précisément parce que cette définition ne nous fournit pas de critère. Je ne peux comparer mon jugement avec l'objet réel que si je connais déjà celui-ci ${ }^{57}$. La seule vérité que contient cette définition est qu'un jugement vrai doit être en harmonie avec les objets, mais ceci est

50. I bid., p. 150, cf. le typoscrit EL 96, p. 5 et suivantes.

51. Brentano, 1889, p. 79 [66].

52. Ibid., p. 79 [66] ; Brentano, 1974, p. 141.

53. Brentano, 1974, p. 157.

54. Cf. Brentano, 1956, p. 165 et suivantes.

55. Cf. Brentano, 1970, p. 150.

56. Brentano, 1889, p. 19, $90[21,80] ; 1974$, p. 156.

57. Brentano, 1974, p. 28. 
trop général pour servir à définir la notion de vérité. Cette harmonien'est pas une correspondance au sens littéral.

Pourquoi Brentano tient-il à confondre les notions de vérité et d'évidence? N e disons-nous pas souvent d'un jugement aveugle qu'il est vrai? Brentano admet qu'un jugement aveugle peut être vrai. Dans la mesure où un jugement n'est pas évident lui-même mais que, en ce qui concerne son contenu, il correspond complètement à une évidence, on peut dire que ce jugement est vrai ${ }^{58}$. M ais nous utilisons alors les mots « vrai » ou « vérité » au sens modifié. "A u sens originel, un "jugement vrai" signifie une évidence ; au sens figuré[in übertragenem Sinne] un jugement aveugle, qui correspond à un jugement évident sous tous les autres aspects, est aussi dit vrai ${ }^{59}$. Le terme "vérité», explique Brentano, est équivoque, ou encore, selon la terminologie de ses écrits antérieurs, " analogue». N ous n'avons de connaissance que quand nous jugeons vraiment selon le premier sens. $N$ éanmoins, la véritéau sens large peut nous aider dans la pratique, comme nous pourrions obtenir des vérités en calculant avec des chiffres qui sont des ersatz des vrais nombres, parce qu'il y a un rapport systématique entre les signes et ce qui est en réal ité.

II n'est pas nécessaire qu'un jugement vrai au sens second corresponde à un jugement évident prononcé actuellement, tant qu'il est possible qu'une personne prononce ce jugement avec évidence. $D$ ans sa difficile définition de 1915, Brentano préfère une formulation en termes de personnes jugeant, conformément à son réisme : la vérité revient au jugement d'un juge correct, c'est-à-dire au jugement de celui qui jugerait comme quel qu'un qui juge avec évidence ${ }^{60}$. Le conditionnel (« jugerait ») est essentiel dans cette définition, et il exprime une modalité. A insi, la vérité d'un jugement ne dépend pas de l'actualité de l'évidence de ce jugement. Un jugement vrai est évidentiable, peut être jugé avec évidence. Dans l'ontologie du « vieux » Brentano, il n'y a pas de place pour les possibilités comme êtres indépendants. Puisque sa théorie de la vérité de cette période n'est pas élaborée complètement, nous devons nous imaginer ce qui s'accorde avec sa théorie: on peut dire qu'un certain jugement est évidentiable quand il est évident pour quelqu'un ou quand on a une méthode qui peut le rendre évident. En ce sens, les possibilités n'existent que pour autant qu'il existe des actualités.

On peut se demander à quoi ressemble la personne idéale capable de juger avec évidence. Certainement, le Brentano des écrits postérieurs croit que toute vérité doit être en principe accessible à la recherche humaine ${ }^{61}$.

58. Q uand on ne veut pas utiliser la notion de contenu, on peut dire : dans la mesure où ce jugement correspond à un jugement évident en acte, qualité et mode.

59. Brentano, 1970, p. 150 ; cf. EL 96, p. 5 (typoscrit 1914).

60. Brentano, 1974, p. 139. Cf. aussi le typoscript EL 45a : « [...] daß die Wahrheit dem Urteile des richtig Urteilenden zukommt, d.h. dessen, der urteilt, wie derjenige darüber urteilen würde, der mit Evidenz sein Urteil ausspricht, also dessen, der das behauptet, was auch der evident Urteilende behaupten würde. »

61. Brentano, 1911, p. 167. Cf. «Bestehen doch für alle erkennenden Wesen dieselben Beweisgründe. »(Brentano, 1974, p. 150). 
Rien d'humain n'est étranger au juge idéal. Pour éclairer cette idée, on peut penser à l'idée du bien comme le conçoit A ristote : ce qui est vraiment un objet de désir est un objet de désir de l'honnête homme ${ }^{62}$. Ici, nous trouvons une différence importante avec l'épistémologie brentanienne antérieure : dans ses écrits postérieurs, il n'est plus possible pour Brentano de prendre Dieu comme juge idéal. Cette théorie de la vérité contient l'idée que tout jugement n'est pas vrai ou faux. $M$ ais Brentano n'en a jamais tiré cette conséquence radicale. Jusqu'à la fin de sa vie il défend explicitement la loi du tiers exclu. Brentano est toujours resté réaliste.

En quel sens peut-on dire que la vérité comme évidence est primaire et que la vérité comme évidentiable est secondaire? Du point de vue de la définition, la vérité comme évidence est primaire, comme on peut le voir dans la définition de la vérité au sens large donnée ci-dessus. N ous ne pouvons saisir ce qu'est un jugement vrai au sens secondaire sans avoir saisi ce qu'est un jugement évident. De plus, nous ne pouvons pas parler d'un jugement aveugle, mais vrai, sans avoir le moyen de rendre ce même jugement évident. En tant que tel, le jugement évident est primaire du point de vue de la connaissance. Q uand Brentano parle de l'usage primaire contrairement à l'usage secondaire du terme de vérité comme de l'usage propre contrairement à l'usage impropre de ce terme, cela ne veut pas dire que l'usage impropre du terme de vérité n'a pas de place dans sa philosophie. En fait, Brentano emploie en général le terme de « vérité » dans le sens secondaire.

Brentano considère les termes de « vérité » et de « correction » comme équivalents ${ }^{63}$ : les deux termes ont la même ambiguïté. Si un jugement est correct à tous les égards, c'est-à-dire s'il est évident, Brentano dit qu'il est justifié logiquement ou qu'il est logiquement correct. Le jugement qui est logiquement correct est tel qu'il doit être, et seulement s'il est tel qu'il doit être, il peut être dit vraiment correct ${ }^{64}$. Un jugement aveugle qui correspond à ce jugement évident à tous les égards est dit « qualitativement correct »65. Dans une lettre à Kraus d'avril 1916, Brentano dit qu'un jugement est correct s'il correspond (« entspricht ») à la règle logique ${ }^{66}$. Il ne faut pas comprendre cette règle comme une proposition ou une valeur objective, isolée des jugements concrets, parce qu'en ce cas, dit Brentano, la correction de cette règle elle-même devrait être garantie $e^{67}$. Q uoi qu'il en soit, cette règle logique indique clairement une composante normative. Le jugement évident est la norme de tous les jugements.

62. A ristote, Ethique à $\mathrm{N}$ icomaque, $1113 \mathrm{a} 52$.

63. Brentano, 1974, p. 131.

64. Brentano, 1966, p. 305.

65. Parfois, Brentano garde le terme «vérité » pour la correction qualitative, et « correction » pour la correction logique; voir les deux lettres à Kraus d'avril et de mai 1916 (Brentano, 1966, p. 300, 305).

66. Brentano, 1966, p. 300 ; voir aussi Brentano, 1889, p. 10[12].

67. Brentano, 1889, p. 81[68]. 
La théorie de la vérité de Brentano n'est pas une théorie de la correspondance. Ce n'est pas non plus une théorie de la cohérence - quoique la cohérence nous aide à saisir la vérité des jugements indirectement évidents. Au mieux on peut la caractériser comme une théorie qui considère la vérité comme une norme, ou comme « rectitude». M oins approprié est le nom classique de " vérité d'objet », que l'on trouve dans les commentaires sur saint Anselme, car Brentano ne semble pas dire que la vérité de l'objet est la norme. Son réisme donne la priorité à la personne qui juge. Pour Brentano, la question est la suivante : comment juge une personne qui juge vraiment correctement? Est-ce que je suis, comme personne jugeant, tel que je dois être? Une certaine « justesse interne », comme dit Brentano, fait que les jugements évidents sont préférables à ceux qui sont non évidents ${ }^{68}$. La vérité se trouve donc dans la correspondance avec quelque chose d'autre qu'un jugement ; elle se trouve dans l'évidence même. Le jugement évident est celui auquel nous aspirons. Le jugement évident est vrai au sens le plus propre. Les jugements non évidents sont eux aussi vrais, mais ils ne le sont que pour autant qu'ils soient mis en relation avec les jugements évidents. En ce sens, il y a une relation systématique entre les sens différents du mot « vérité », de sorte que ce terme est analogue par référence à I'Un - la forme d'analogie que Brentano a rencontrée dans la pensée d'A ristote et dans la scolastique. La vérité comme évidence est le sens auquel les autres sens de la vérité sont référés comme à l'Un, comme au terminus.

\section{Bibliographie}

Aristote, Categories and D e Interpretatione, Ackrill, J.L., dir., O xford, 1979 - - , N icomachean Ethics, dans Barnes, J., dir., The Complete Works of A ristotle, Princeton, 1991

- - , Posterior Analytics, Loeb, dir., Cambridge, London, 1989

- - , M etaphysics, Loeb, dir., Cambridge, London, 1977

Bolzano, Bernard W issenschaftlehre, Sulzbach, 1837

Brentano, Franz, D ie Abkehr vom N ichtrealen, M ayer-H illebrand, F., dir., Bern/M ünchen, 1966

- - , Vom D asein G ottes, Kastil, A., dir., H amburg, 1980

- - , Versuch über die Erkenntnis, Kastil, A. et M ayer-H illebrand, F., dir., H amburg, 1970

_- , Wahrheit und Evidenz, Kraus, O skar, dir., Hamburg, 1974

- - , Vom U rsprung sittlicher Erkenntnis, Leipzig, 1889; et idem, Kraus, O skar, dir., H amburg, 1969 (la pagination de cette édition est donnée entre crochets)

- - , «L'origine de la connaissance morale (1889) », traduit par M arc B. de Launay, Revue de M étaphysique et de M orale, 1, 1990, p. 3-32

68. Ibid., p. 11[13]. 
_- , Psychologie vom empirischen Standpunkt, Leipzig, 1874

_- , Von der Klassifikation der psychischen Phänomene, Leipzig, 1911

- - , D ie L ehre vom richtigen U rteil, M ayer-H illebrand, F., dir., Bern, 1956

- - Von der mannigfachen Bedeutung des Seienden nach Aristoteles (1862), H ildesheim, 1960

- - , Kategorienlehre, Kastil, A., dir., $\mathrm{H}$ amburg, 1985

_- , A ristoteles und seine W eltanschauung (1911), H amburg, 1977

Chisholm, Roderick M., " Correct and Incorrect Emotion », Brentano and M einong Studies, A msterdam, 1982

- - , Brentano and Intrinsic Value, Cambridge, 1986

Gens, J ean-Claude, «La doctrine du jugement correct dans la philosophie de F. Brentano », Revue de M étaphysique et de Morale, 3, 1996, p. 361381

H usserl, Edmund, Briefwechsel, Bd. I, die Brentanoschule, Schuhmann, E. et Schuhmann, K., dir., D ordrecht, 1994

Kahn, Charles H., "Questions and Categories», dans Hiz, H., dir., Q uestions, Reidel, 1978, p. 227-278

M elandri, Enzo, «The "Analogia Entis " according to Franz Brentano », Topoi, 6, 1987, p. 51-58

Lyttkens, $\mathrm{H}$., The A nalogy between $\mathrm{G}$ od and World ; An Investigation of its Background and Interpretation of its $U$ se by Thomas of Aquino, Uppsala, 1952

O wen, G.E.L., "Logic and Metaphysics in Some Earlier Works of A ristotle », dans Düring, I., O wen, G.E.L., dir., A ristotle and Plato in the M id-Fourth Century, Göteborg, 1960, p. 163-190

Saint Anselme, O ver waarheid / D e Veritate, Vanderjagt, A., dir., Kampen, 1990

Saint Thomas d'A quin, $O$ ver waarheid en onwaarheid/D e veritate et fal sitate (Summa Theologiae, I, questions 16 et 17), te Velde, R.A., dir., Kampen, 1988

Saint Thomas d'Aquin, Von der Wahrheit/De Veritate (Q uestio I), Zimmerman, Albert, dir., H amburg, 1986

Klubertanz, G.P., St. Thomas A quinas on A nalogy ; A Textual A nalysis and Systematic Synthesis, Chicago, 1960

Paul Wilpert, "Zum A ristotelischen Wahrheitsbegriff », dans H ager, F.-P., dir., Logik und Erkenntnislehre des Aristoteles, Darmstadt, 1972, p. $106-121$ 